\title{
Accuracy of an intelligent CPAP machine with in-built diagnostic abilities in detecting apnoeas: a comparison with polysomnography
}

\author{
Matthias Gugger, Johannes Mathis, Claudio Bassetti
}

\begin{abstract}
Background - In patients with sleep apnoea early diagnostic evaluation and treatment may be delayed due to limited access to full polysomnography (PSG). For "typical" patients, simplified strategies are needed. A study was performed to evaluate the accuracy of a new continuous positive airway pressure (CPAP) device with in-built diagnostic abilities (Autoset) in detecting apnoeas.
\end{abstract}

Methods - Twenty seven patients underwent full overnight polysomnography. Data with the Autoset were acquired simultaneously. Standard nasal prongs were used. Apnoeas were detected by special analysis of the flow signal. As the Autoset derives all its data from one signal, careful examination of the raw data is important to assess the quality of the flow signal.

Results - There was a correlation between the apnoea index (AI) assessed by the Autoset (AI-Autoset) and by polysomnography AI-PSG $(r=0 \cdot 85)$ and between the AI-Autoset and the apnoeal hypopnoea index (AHI) during polysomnography $(r=0 \cdot 87)$. The Autoset identified patients with an AHI-PSG of $>20$ (a level of respiratory disturbance that would warrant consideration for treatment in most centres for sleep disorders) with a sensitivity of $82 \%$ and a specificity of 90\%.

Conclusions - The good correlation between the apnoea index measured by the Autoset and by polysomnography, and the high sensitivity in detecting patients with an AHI of $>20$, may make the Autoset a valuable tool for the management of typical patients with sleep apnoea. However, very low values for nasal ventilation on the printout raises the suspicion of poor signal quality and misleading results.

(Thorax 1995;50:1199-1201)

Keywords: automatic apnoea detection, sleep apnoea, intelligent CPAP, polysomnography, Autoset.

Obstructive sleep apnoea is a common and debilitating condition affecting up to $6 \%$ of the population. ${ }^{12}$ Nasal continuous positive airway pressure (CPAP) is an effective treatment and the first choice therapy. ${ }^{34}$ Polysomnography is regarded as the "gold standard" for the diagnosis of sleep apnoea and review sleep studies with CPAP. However, polysomnography is a labour and time intensive method and many patients are awaiting sleep studies. The need for full polysomnography delays the start of treatment and reassessment where required. There is still no generally accepted cheaper method for the management of the "typical" patient with sleep apnoea. ${ }^{4-7}$ The Sullivan Autoset (ResCare Ltd, Sydney, Australia) offers a new approach to the management of patients with a typical case history and clinical findings. ${ }^{7}$ The costs of an Autoset study are estimated to be several times lower than for full polysomnography, although there are no generally agreed prices for both investigations in Switzerland. It was beyond the scope of this report to assess the effectiveness of treatment with the Autoset, but we aimed to validate its inbuilt diagnostic abilities for detecting apnoeas. The diagnostic accuracy was determined by comparison with the results of simultaneous polysomnography.

\section{Methods}

A computer controlled nasal CPAP flow generator (Sullivan Autoset) with the prototype software "Autumn 1994" was used. This device is designed for computer controlled CPAP titration (treatment mode). However, it can also be used to assess the severity of the respiratory disturbance at night and for this application the diagnostic mode is selected. An oximeter and standard nasal prongs (instead of a nasal mask) are the only sensors needed. Nasal airflow is measured semiquantitatively (arbitrary units, range $0-20) .^{78}$ The nasal cannulae are connected to a pressure transducer in the Autoset device. The pressure versus nasal flow relationship is then linearised with software. The manufacturers claim that, although the magnitude of the signal will depend on the fit between the cannulae and the nostrils, relative changes are measured with an accuracy of $\pm 10 \%$ in the range $0 \%$ to $150 \%$ control. An apnoea corresponds to cessation or near cessation of nasal airflow for 10 seconds or 
Figure 1 Correlation (AI) assessed with the Autoset (AI Autoset) and the $A I$ assessed by polysomnography (AI PSG). between the apnoea index

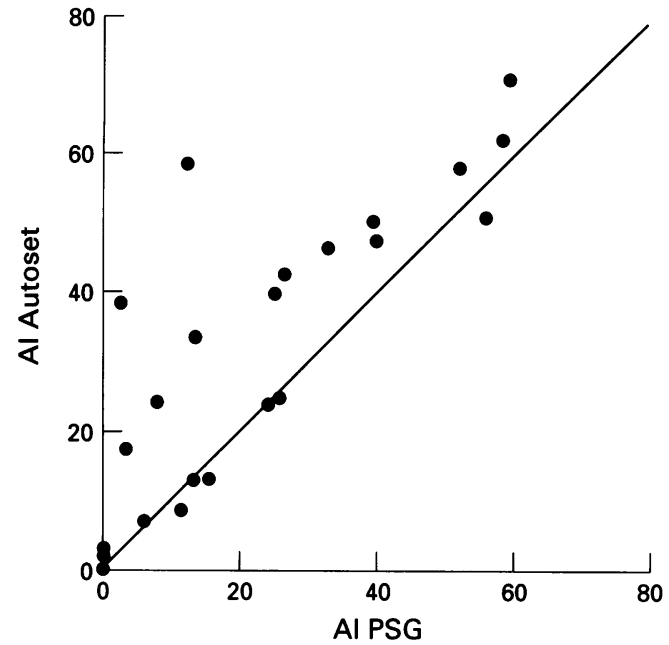

more. The apnoea index is the number of apnoeas/hour of recording. The snore signal is derived by bandpass filtering the flow signal $(30-300 \mathrm{~Hz})$ and adjusting for background motor noise. It is expressed in arbitrary units with one unit representing a "typical" snore. The flattening index is a measure of the root median square deviation from the normalised mean inspiratory flow rate over the middle $50 \%$ of inspiratory time. A value of zero would indicate a square wave flow-time profile. The manufacturers state that a normal awake value exceeds 0.25 units, and the median value in all night studies on 16 normal subjects was 0.15 units. The current software was not designed for detection of hypopnoeas.

Twenty seven consecutive patients (four women) of median age 51 years (range 19-71), median body mass index $29 \mathrm{~kg} / \mathrm{m}^{2}$ (range 19-43), and median Epworth score 10 (range 1-18) underwent standard overnight polysomnography in our sleep laboratory. Their final diagnosis was obstructive sleep apnoea syndrome in 18, persistent sleep apnoea after uvulopharyngopalatoplasty in one, simple snoring in one, familial sleep paralysis in one, hypersomnia with mood disorders in two, narcolepsy in one, insufficient sleep syndrome in one, thalamic stroke in one and Prader-Willi syndrome in one. Two electroencephalograms, two electro-oculograms, electromyograms (submental, left and right tibial), oronasal flow

Figure 2 Difference between the apnoea index (AI) assessed with the Autoset and by polysomnography (PSG) plotted against the mean apnoea index (Bland-Altman plot).

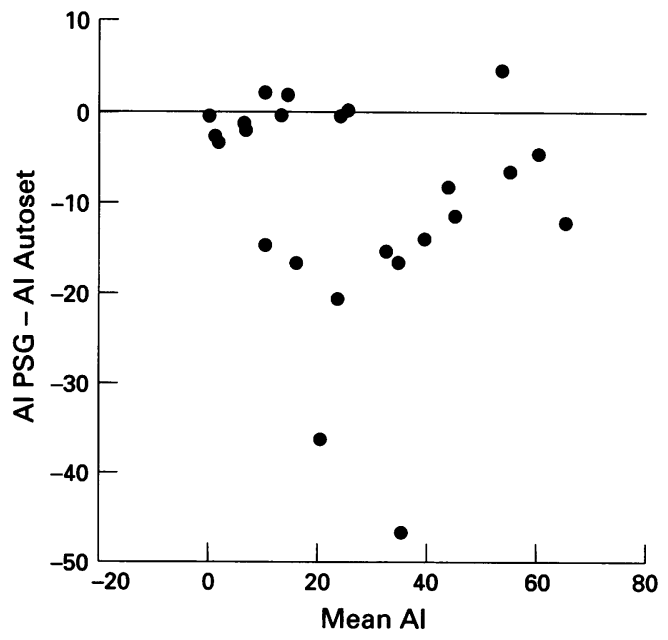

(thermistors), thoracoabdominal movements (respiratory inductive plethysmography, Respitrace), electrocardiography and position were recorded. The oximeter was connected both to the polygraph (Nihon Kohden) and the Autoset; the nasal prongs to the Autoset. All recordings were made simultaneously. On-line display of the Autoset flow curve and data and an infrared video system with zoom facilities and a microphone allowed continuous supervision by the sleep technician. Standard criteria were used for the analysis of the polysomnography data. ${ }^{9}$ After each recording night an Autoset report was generated which provides an apnoea index, graphic information on oxygen saturation, snoring, nasal ventilation (arbitrary units $0-20$ ), the flattening index, and the number of apnoeas (indicating each apnoea and its duration) throughout the study, with several summary graphs of these data. ${ }^{4}$ The software offers no editing facilities and the data have to be accepted as they are.

\section{Results}

Figure 1 shows the correlation between the apnoea index (AI) derived from standard polysomnography and the apnoea index assessed with the Autoset $(r=0 \cdot 85)$. The Bland-Altman plot (fig 2) shows a small tendency for overscoring by the Autoset. There was also a significant correlation between the apnoea/ hypopnoea index (AHI) derived from polysomnography and the $\mathrm{AI}$ assessed with the Autoset $(r=0 \cdot 87)$. The correlation between the hypopnoea index (HI) derived from polysomnography and the $\mathrm{AI}$ assessed with the Autoset was poor $(r=0 \cdot 48)$. The sensitivity and specificity of the Autoset in detecting patients with an AHI of $>20$ during standard polysomnography was $82 \%$ and $90 \%$, respectively, and of oximetry (>20 $4 \%$ oxygen dips) was $76 \%$ and $69 \%$, respectively. Figure 3 shows the relationship of the difference between AI assessed by polysomnography and by the Autoset, and the level of ventilation.

\section{Discussion}

The main finding of this study is the good correlation between the AI assessed with an intelligent CPAP machine with in-built diagnostic abilities (Autoset) for detecting apnoeas and the AI derived from simultaneous polysomnography (fig 1). However, there was a small tendency for the Autoset to overestimate the AI (fig 2). Although the Autoset software used was designed for apnoea detection, the correlation of the Autoset-AI with the combined apnoea and hypopnoea index (AHI) from polysomnography was equally good.

Our results were obtained under ideal conditions in a sleep laboratory with a sleep technician. Although handling of the Autoset is easy, false positive or negative results may be obtained due to problems including nasal prong dislodgement or blockage by secretions, especially during unattended studies or if the patient predominantly mouth breathes. As the Autoset derives all its data from one signal, it is par- 
Figure 3 Relationship between the difference between the apnoea index assessed with the Autoset and by polysomnography (PSG) and the percentage time of nasal ventilation below 5 arbitrary units (range 0-20).

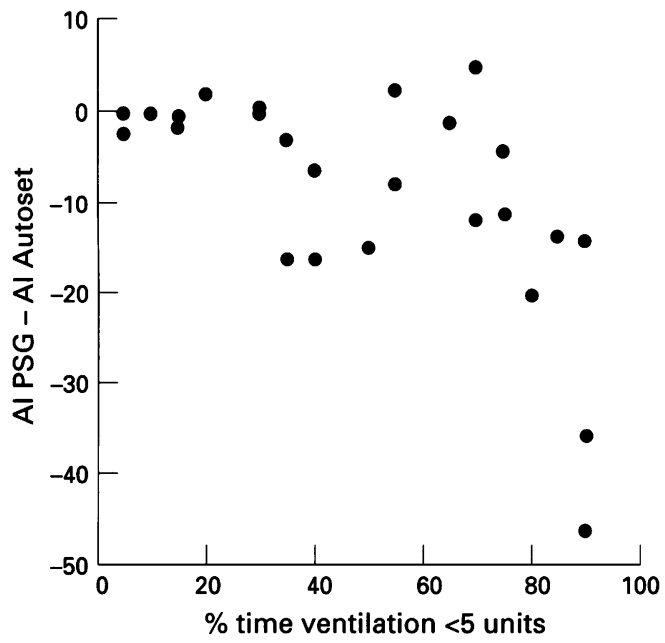

ticularly important to examine the raw data to assess the adequacy of the flow signal. A very low value for nasal ventilation on the report printout, for example, raises the suspicion of poor signal quality. Figures 1 and 2 show two outliers. Figure 3 shows that in both cases nasal ventilation was very low ( $<5$ arbitrary units) for $>90 \%$ of the study time (one outlier represents the patient after uvulopalatopharyngoplasty, one a patient with uncomplicated sleep apnoea). These points stress the importance of considering the complete data set of the report and not just the final number for the AI.

The Autoset is not designed to replace full diagnostic or therapeutic evaluation, but to provide adequate, although in some cases possibly provisional, care for the typical patient with sleep apnoea. The sensitivity and specificity of the method were therefore calculated for an AHI-PSG of $>20$, a level of respiratory disturbance that would warrant treatment in most sleep centres. ${ }^{1011}$ The sensitivity of $82 \%$ and the specificity of $90 \%$ show that a reasonable number of patients can be managed by this strategy as a first step, and that the Autoset did better in these patients than oximetry alone ( $76 \%$ and $69 \%$, respectively). Some caution is needed with the values for sensitivity and specificity because of the relatively small numbers of patients studied. Furthermore, these values also indicate that, in patients with a clinical suspicion of sleep apnoea but negative Autoset results, or positive Autoset results but unsatisfactory response to treatment, or whenever the results are ambiguous, full overnight polysomnography must be performed.

1 Partinen M. Epidemiology of sleep disorders. In: Kryger MH, Roth T, Dement WC, eds. Principles and practice of sleep medicine. 2nd ed. Philadelphia: WB Saunders, 1994 437-52.

2 Young T, Palta M, Dempsey J, Skatrud J, Weber S, Badr S. The occurrence of sleep-disordered breathing among middle-aged adults. $N$ Engl $\mathcal{F}$ Med 1993;328:1230-5.

3 Sullivan CE, Issa FG, Berthon-Jones M, Eves L. Reversal of obstructive sleep apnea by continuous positive airway pressure applied through the nares. Lancet 1981; arwa

4 pressure applied through the nares. Lancet 1981;1:862-5. agement of obstructive sleep apnoea/hypopnoea synagement of obstructive sleep
drome. Lancet 1994;344:656-60.

5 Miles LE, Buschek GD, McClintock DP, Miles SC, Narvios LR, Wang YX. Development and application of automatic nasal CPAP calibration procedures for use in the unsupervised home environment. Sleep 1993;16:S118-19.

6 Chediak AD, Lipson E, Cuneyt Demirozu M, Kiel M. The second generation of nasal continuous positive airway pressure devices. Are they created equal? Sleep 1993;16: 662-7.

7 Berthon-Jones M. Feasibility of a self-setting CPAP machine. Sleep 1993;16:S120-3.

8 Condos R, Norman RG, Krishnasamy I, Peduzzi N, Goldring RM, Rapport DM. Flow limitation as a noninvasive assessment of residual upper airway resistance during continuous positive airway pressure therapy of obstructive sleep apnea. Am F Respir Crit Care Med 1994;150:475-80

9 Gould GA, Whyte KF, Rhind GB, Airlie MAA, Catteral JR, Shapiro CM, et al. The sleep hypopnea syndrome. Am Rev Respir Dis 1988;137:895-8.

10 Kryger MH. Management of obstructive sleep apnea. Clin Chest Med 1992;13:481-92.

11 Phillipson EA. Sleep apnea, a major public health problem. $N$ Engl f Med 1993;328:1271-3.

\title{
Comparison of polysomnography with ResCare Autoset in the diagnosis of the sleep apnoea/hypopnoea syndrome
}

\author{
Pauline A Bradley, Ian L Mortimore, Neil J Douglas
}

\footnotetext{
Respiratory Medicine Unit, Department of Medicine, Royal Infirmary, Edinburgh EH3 9YW, UK P A Bradley I L Mortimore N J Douglas

Correspondence to: Professor N J Douglas.

Received 23 February 1995 Returned to authors 5 June 1995 Revised version received 20 July 1995 Accepted for publication 24 July 1995
}

\author{
Abstract \\ Background - Increasing referral num- \\ bers make the development of simplified \\ accurate methods of diagnosing the sleep \\ apnoea/hypopnoea syndrome highly de- \\ sirable. The accuracy of one such system - \\ the ResCare Autoset - has been examined. \\ Methods - Thirty one consecutive patients \\ assessed by polysomnography had sim- \\ ultaneous monitoring of their respiratory \\ pattern using the Autoset system. The \\ Autoset detects episodes of flattening of
}

the flow/time profile using nasal cannulae. Results - There was a good correlation $(r=0.85)$ between the number of apnoeas + hypopnoeas/hour in bed recorded using polysomnography and the Autoset system. The median difference in such events was $3 \cdot 1 \quad(95 \%$ confidence interval 8.4 to $-1 \cdot 6$ )/hour in bed. In two patients the Autoset scored 70 apnoeas + hypopnoeas/hour in bed compared with 34 apnoeas + hypopnoeas with 35 arousals/ hour in bed by polysomnography; how- 